\title{
OPTIMAL POSITIONING METHODS OF INTEGRAL DEFORMATION SENSORS - EXPERT KNOWLEDGE VERSUS MATHEMATICAL OPTIMIZATION
}

\author{
Ch. Brecher ${ }^{1,2}$, R. Herzog ${ }^{3}$, A. Naumann ${ }^{3}$, R. Spierling ${ }^{1 *}$, F. Tzanetos ${ }^{2}$ \\ ${ }^{1}$ RWTH Aachen University, Laboratory for Machine Tools and Production Engineering (WZL), Aachen, Germany \\ ${ }^{2}$ Fraunhofer Institute of Production Technology (IPT), Aachen, Germany \\ ${ }^{3}$ TU Chemnitz, Faculty of Mathematics, Chemnitz, Germany \\ *Corresponding author; e-mail: R.Spierling@wzl.rwth-aachen.de
}

\begin{abstract}
Up to $75 \%$ of the overall work piece error can be caused by the thermo-elastic behavior of the machine tool. Therefore, correction methods based on machine-integrated sensors were intensively researched during the last years, in order to determine the error of the Tool Center Point (TCP) parallel to the process. One of these methods includes the integral deformation sensor (IDS), which detects the deformation along the length of a structural component of the machine. The error of the TCP is modelled based on the measured structural deformations, a mechanical model of the structural parts and a kinematic model of the machine tool.

Currently, the sensor setup for specific machines is usually defined by an expert with the help of his or her domain knowledge. There are existing mathematical methods for optimal sensor positioning. The aim of this work is the evaluation of the expert positioning versus the mathematical methods. The parameters to be varied are the lengths and positions of the IDS. Criteria for the evaluation are the achievable accuracy of the TCP error prediction and the sensitivity to small variations of the optimal position, as they might occur during the installation.
\end{abstract}

\section{Keywords:}

Thermal issues; machine tool; optimal sensor positioning;

\section{INTRODUCTION}

The majority of research and industry has focused on temperature sensors as input variables for thermal error compensation in machine tools. Although some approaches make use of Fiber-Bragg strain sensor networks, CNC-internal data like motor currents or even gather additional intermittent measurements of the TCP error, optimal sensor positioning methods have been researched only for temperature sensors. The diversity of physical and phenomenological modeling methods has led to an analogous diversity of optimal sensor positioning methods.

Predominantly, experimental data and experience from trial-and-error have been the base of sensor positioning methods, both in industry and in research. This information is then fed into statistical and phenomenological models that strive to find the optimal correlation between TCP error and temperature sensors. In their simplest form, such methods include regression analysis [Liu 2020]. More complex and sophisticated phenomenological methods include fuzzy clustering and grey correlation modeling [Wei 2018], density peaks clustering [Zhou 2019] or nature-inspired optimization like the binary bat algorithm [Tan 2020]. As a result, all methods are dependent on the effort invested to run as many and as diverse loading cases as possible. In industry, only large (in business size) machine tool manufacturers have been able to run experimental investigations for the purpose of identifying the optimal temperature sensor positioning. On the other hand, smaller manufacturers rely on experienced machine tool designers and simplified thermo-mechanical FEM simulations based on the power specifications of the drive components.

The integral deformation sensors (IDS) can significantly contribute to the determination of the thermo-elastic behavior of machine tools and subsequently the precision of the manufactured parts. A physical model, based on mechanical modelling and the kinematics of the machine tool, calculates the Tool Center Point (TCP) error in realtime parallel to the machining process [Brecher 2018].

The determination of the optimal sensor positioning can reduce the amount of sensors needed to reach the same prediction accuracy. This work concentrates on the reduction of the uncertainty propagation from the measurement uncertainty of the IDS data to the predicted TCP error. The sensor measurement can be seen as an 
experiment. Hence, the optimal sensor positioning is an example of an optimal experimental design (OED). The review [Atkinson 1996] showed, that the OED methods are quite successful in several disciplines.

There are two possible applications for the IDS system. Either the system is applied to a machine in the context of a new design where there are extensive degrees of freedom to enable an optimal positioning of the system. Or the IDS system is retrofitted to an existing machine. In this case, the choice of possible mounting surfaces is limited by the existing construction. This work introduces the mathematical fundamentals for both cases, but focusses on the retrofit in the measurement results section.

The performance of the system with regard to the correction of thermo-elastic errors is optimized here with respect to two factors. These are the prediction accuracy of the model and the measurement uncertainty of the errors. Both factors are significantly influenced by the positioning of the sensors.

Therefore, the aim of this paper is to compare different optimization methods for IDS positioning. In section 2 the TCP error prediction based on IDS is presented and an experience-based optimization method is compared with a mathematical black-box optimization method. The mathematical approach developed in [Brecher 2020] was extended for this purpose to enable the evaluation of the error in the work space. Results are presented for a design phase implementation of IDS and a retrofit of IDS. Chapter 3 presents a first experimental validation of different calculated measurement setups. The summary and the outlook in chapter 4 conclude this work.

\section{METHODOLOGY}

Where to place a sensor, and what quantities should be measured are important questions to directly observe the machine behavior. In general the direct measurement of the TCP error is not feasible, therefore the measurement of displacements or other quantities is suitable, as every measurement requires a model using the measured values as inputs in order to approximate the TCP error.

Every model is based on assumptions. In this case the simplification of the machine structure and homogeneous material properties for all components are assumed. These assumptions are the main source of model errors. Consequently, the computed TCP error is always slightly perturbed, even in the case of hypothetically exact measurements.

Every model also contains parameters. These may include material parameters, or assumptions on them, and in our case, the sensor positions are also part of the model parameters.

At the same time a sensor is also not perfect. As a result, the actual model input is always a superposition of the nominally measured value and a measurement error. These measurement errors are part of the model calculations, hence it is a property of the model whether these measurement errors are amplified, damped or stay in the same in magnitude.

The paragraph 2.1 explains the modelling of the TCP error based on the IDS inputs. This is the so called prediction model. The subsequent paragraphs 2.2 and 2.3 explain two approaches to obtain the optimal sensor positioning in two different scenarios. First the positioning based on expert knowledge is discussed. Second approach uses mathematical tools to classify and optimize the positioning.

Both approaches can be applied to new machine designs and retrofit of an existing machine.

\subsection{Integral deformation sensors and modeling of the thermally induced TCP error}

The measuring principle of the IDS for the detection of thermally induced TCP errors in machine tools relies on reference rods made of carbon-fiber reinforced plastic (CFRP) that are mounted on the surfaces of the passive machine tool structure. Since a machine tool performs a relative motion between tool and work piece, components can be categorized as active components performing such motions, e.g. guides and driving systems, and passive components, such as a machine bed and a moving column, whose purpose is to withstand the loads during the manufacturing process. Using an appropriate displacement transducer, the relative elongation in the direction of the reference rod can be measured. Taking into account that CFRP features a very low thermal expansion coefficient of down to $-0.1 \mu \mathrm{m} / \mathrm{m} / \mathrm{K}$, depending on the direction of the carbon fibers, the detected elongation is characteristic for the deformation of the machine tool structure in the direction of the reference rod.

With a sufficient number of well-positioned IDS and with a suitable deformation model, it is possible to determine the displacement field of the machine tool structure. An example to explain the combination of several IDS is a two-dimensional beam, supported on one side. In case of bending due to thermal load, the beam fibers on the one side of the neutral fiber will expand, while the beam fibers on the other side will contract. An IDS on the expanding beam fibers and an IDS on the contracting beam fibers can thus identify the bending inclination and deflection of the beam.

In detail, assuming linear material properties, a machine component expands proportionally to the temperature difference in the longitudinal direction. The change in the $x$ dimension $\Delta l_{x}$ is dependent on the time $t$, the coordinate $x$, the coefficient of linear thermal expansion $\alpha$, the temperature change in $x \Delta T_{x}$ and the initial length $l_{0}$, see Eq. 1 and Fig. 1.

$\Delta l_{x}(t, x)=l_{x}(t, x)-l_{x}(t=0, x)=\alpha \cdot \Delta T_{x}(t, x) \cdot l_{0}$

The proportionality factor depends on the initial length $l_{0}$ and the thermal expansion coefficient $\alpha$ of the material of the machine tool structure, which is usually mineral casting or cement concrete based composite $(11 \mu \mathrm{m} /(\mathrm{m} \bullet \mathrm{K}))$. It is evident here, that the CFRP reference rod mounted in the direction of elongation expands less than the machine tool structure. However, the temperature gradient can also show a linear, quadratic or arbitrary behavior, so the temperature profile is integrated over the component length $l_{0}$, see Eq. 2 .

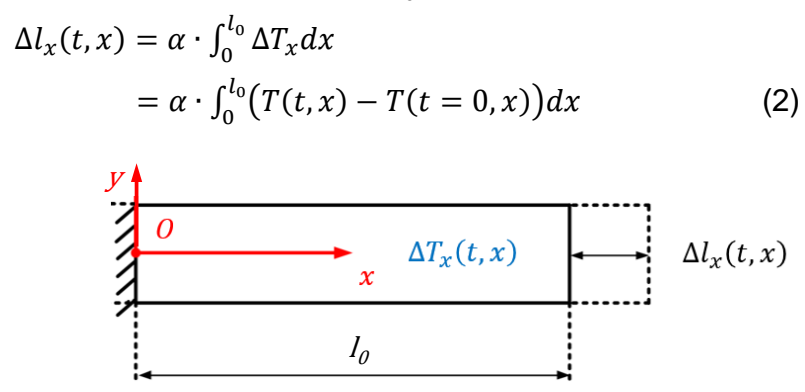

Fig. 1: A simple example of linear expansion due to a longitudinal temperature profile 
Consequently, the IDS hence measure directly the left side of the aforementioned equation, which is defined by the authors as integral deformation.

In addition, a temperature profile along the cross section of a machine tool component $\Delta T_{y}$ leads to two further deformations. The bending inclination $\theta(x)$ and the bending deflection $\Delta y$. Both values depend on the height of the cross-section $H$, see Eq. 3 and 4 as well as Fig. 2 .

$$
\begin{aligned}
\Delta y(x) & =\int_{0}^{l_{0}} \theta(x) d x=\alpha \cdot \iint_{0}^{l_{0}} \frac{T\left(y_{2}\right)-T\left(y_{1}\right)}{y_{2}-y_{1}} d x^{2} \\
& =\alpha \cdot \frac{\Delta T_{y}}{H} \cdot \frac{l_{0}^{2}}{2}
\end{aligned}
$$

$$
\begin{aligned}
\theta(x) & =\int_{0}^{l_{0}} d \theta(x) d x=\int_{0}^{l_{0}} \frac{d l_{x}\left(y_{2}\right)-d l_{x}\left(y_{1}\right)}{y_{2}-y_{1}} d x \\
& =\alpha \cdot \int_{0}^{l_{0}} \frac{T\left(y_{2}\right)-T\left(y_{1}\right)}{y_{2}-y_{1}} d x=\alpha \cdot \int_{0}^{l_{0}} \frac{\Delta T_{y}}{H} d x=\alpha \cdot \frac{\Delta T_{y} \cdot l_{x}}{H}
\end{aligned}
$$

If two IDS are positioned on both sides of the neutral fiber of the beam as described above, such deformations can also be mapped. In this case, the height of the crosssection $H$ influences the proportionality factor, i.e. the higher the cross-section, the smaller is the bending for a given temperature profile.

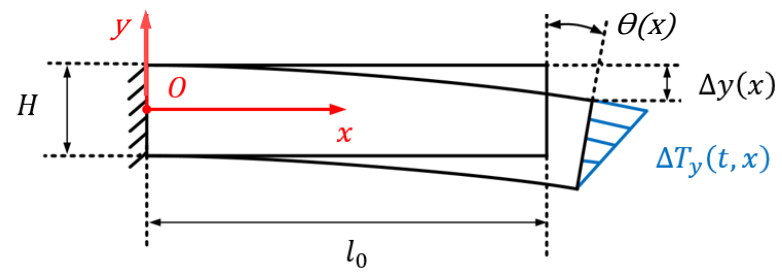

$\Delta T_{y}(t, x)$

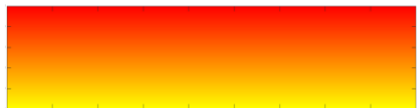

Fig. 2: A simple example of bending due to a crosssectional temperature profile

Therefore, each additional IDS, depending on the overall sensor positioning, can measure directly or determine indirectly an additional degree of freedom for the total deformation of the machine tool component. For example, with IDS arranged in parallel, structures that allow the assumptions of the Euler-Bernoulli beam theory can be mapped with a one-dimensional beam model, for which all further degrees of freedom can be neglected. Most of the machine components in milling, turning and drilling machining centers meet these assumptions because they have one significantly longer dimension.

Positioning additional IDS perpendicular to those for an Euler-Bernoulli beam in one additional direction, twodimensional plate models according to the Love-Kirchhoff assumptions can map the deformation field of the machine tool component accurately. Another use of additional perpendicular IDS is the detection of shear deformations in case that only the Timoshenko assumptions can be met for a beam or the MindlinReissner assumptions for a plate.

Nevertheless, it is very important to note that the accurate application of such models also requires a reliable definition of the mechanical boundary conditions of the machine tool components. For example, the same IDS measurements can lead to a different deformation field for a beam supported on one side in comparison to a cantilever beam. This however is also the reason why the deflection along the beam is partly known before taking the IDS into consideration. The mechanical boundary conditions allow only a pre-defined set of deflection curves, which vary depending on how the thermal load influences the structure. Consequently, the task for the IDS and their positioning is closely related to the mechanical boundary conditions, such as the fixation (clamped beam or simply supported beam) and free stress, of each machine tool component.

\subsection{Experience based optimal positioning}

The combined measurement uncertainty of the IDS amounts $1,3 \mu \mathrm{m} / \mathrm{m}$ [Brecher, 2018]. It is important to note that the measurement uncertainty is dependent on the length of the IDS, since it is partly determined by the true thermal expansion of the used CFRP reference rod. The first guideline is therefore to keep the IDS length as low as possible.

On the other hand, the measurement principle described in section 2.1 stipulates that the IDS measure the integral deformation along their length. This means that the IDS signal contains information about the integral of the temperature profile between its two mounting points. The deformation outside of this area is thus determined indirectly, based on the overall deflection curve permitted by the mechanical boundary conditions. Therefore, the second guideline dictates that the indirectly determined part of the deflection curve should be also kept at minimum.

It is hence evident that the experience based optimal positioning constitutes a compromise between these two guidelines. However, the main goal of this approach is a reliable prediction accuracy. The second guideline is therefore prioritized in this approach, as it improves the model robustness.

A simple example can illustrate the reasoning behind this priority setting. It is common in conventional, middle-sized machine tools to have axes longer than $1 \mathrm{~m}$. In order to gather information through the entire travel length of the machine axes, at least one of the IDS should be longer than $1 \mathrm{~m}$. This automatically leads to at least one input of the prediction model having a measurement uncertainty of more than $1.3 \mu \mathrm{m}$.

Previous theoretical investigations have shown, that the propagation of this uncertainty to the TCP error can be amplified significantly through the lever arms of the rotational error component of the machine tool axes [Riedel 2017]. However, the uncertainty propagation through the translational error components, such as the positioning error or the straightness error, has shown no significant amplification in several machine tool kinematics. Depending on the machine tool size and kinematics, the $2 \sigma$ combined uncertainty of the TCP error prediction from translational error components was smaller than 5-10 $\mu \mathrm{m}$.

As a result, the experience based optimal positioning can lead to a fast and reliable prediction accuracy based on the given constraints of the available installation space on the machine tool structure, the mechanical boundary conditions of each component and the amplifying lever arms of the machine tool kinematics. However, a fast estimation of the optimal sensor positioning requires experience with the commissioning and modeling of the IDS and a reliable definition of the mechanical boundary conditions of the machine tool components.

These two conditions often contradict each other, since information about the mechanical boundary conditions of the machines are not always available. It is common to have little information about the true mechanical constraints of the real structure, which in turn limits the full potential of the IDS. Since both positioning approaches 
are based on the prediction model, this fact limits them as well.

\subsection{Mathematical approach for optimal positioning}

The mathematical approach developed in [Brecher 2020] was extended here to enable the evaluation of the error in the work space. The mathematical approach is based completely on the model properties and the machine geometry. Therefore the model is briefly described and the optimization criteria is derived from a statistical point of view.

The model approximates the component deformation in local coordinates and combines the local deformations through a kinematic chain to the global displacements. The model is the linear equation, see Eq. 5.

$U_{\{T C P\}}\left(x_{I}\right)=\boldsymbol{A}_{\boldsymbol{i}} * s$,

where $U_{\{T C P\}}\left(x_{I}\right)$ represents the approximated TCP error in the point $x_{I}$. The matrix $A_{i}$ is the coefficient matrix of the linear system and contains all model parameters. These coefficients depend particularly on the machine pose as well as the sensor positions. From a statistical point of view, independent normally distributed sensor values are considered, see Eq. 6 .

$s=N(\bar{s}, \Sigma)$

with mean $\bar{s}$ and diagonal covariance matrix $\Sigma$. Furthermore the sensors' covariance depends linearly on the sensor lengths. Thus the covariance of the TCP error depends indirectly on the sensor lengths via the model coefficients $A_{i}$ and additionally the sensor covariance matrix $\Sigma$, see Eq. 7 .

$C_{T C P}\left(x_{i}\right)=A_{i}^{T} \Sigma A_{i}$

The point $x_{i}$ is fixed. The covariance matrix $C_{T C P}$ defines the shape of confidence ellipsoids. Thus the roots of the eigenvalues $\lambda_{i, 1}$ of $C_{T C P}\left(x_{i}\right)$ describe the lengths of the confidence ellipsoid's semi-axes, and the eigenvectors determine the axes' directions. In analogy to [Atkinson 2007] the three optimality criteria are defined

1. The A-criterion considers the average squared axis length, see Eq. 8.

$\frac{1}{3}\left(\lambda_{i, 1}+\lambda_{i, 2}+\lambda_{i, 3}\right)$

2. The $D$-criterion uses the determinant and it can be interpreted as a measure for the confidence ellipsoid's volume, see Eq. 9.

$\operatorname{det}\left(C_{T C P}\left(x_{i}\right)\right)=\lambda_{i, 1} \lambda_{i, 2} \lambda_{i, 3}$

3. The E-criterion uses the maximal (squared) axis length, see Eq. 10.

$$
\max \left(\lambda_{i, 1}, \lambda_{i, 2}, \lambda_{i, 3}\right)
$$

Please note that the $A$ - and D-criterion define a differentiable function, whereas the E-criterion is not differentiable when two axes have the same length. Nevertheless the E-criterion is chosen to minimize the maximal amplification of the measurement errors.

At this point, a criterion for the optimal sensor positioning for one point $x_{i}$ in the work space is defined. The next step includes the whole work space, which renders the optimized positioning robust against movements of the TCP. For the presented case this means that all possible positions of the TCP are minimized concerning the worst result. The work space is therefore approximated with ten points for all coordinates. Afterwards the eigenvalues $\lambda_{i, 1}$ $\ldots \lambda_{i, 3}$ are stacked in one $10^{3} * 3$ sized vector. The optimization aims at minimizing the maximum of all eigenvalues.

The optimal sensor positioning is a constrained optimization problem, where the maximum of the eigenvalues is the objective function. The constraints of the optimization problem are the allowed lengths and positions of the sensors. This work focusses on the case, where all sensors are placed at the machine's surface.

The next two sections describe the different constraints from the scenarios and the consequences for the optimization approach. Both scenarios were implemented in the optimization toolbox IpOpt [Wächter 2002]. The gradients of the eigenvalues were approximated with a second order finite difference stencil and the Hessian was approximated using the limited memory hessian approximation formula BFGS (Broyden-FletcherGoldfarb-Shanno) [Fletcher 2013].

\subsection{Positioning during the design phase}

In the machine's design phase, the designer defines the accessible parts of the machine surface. Several load cases, and their consequences for the work space, can be considered with numerical simulations. These load cases determine the model accuracy and therefore the efficiency of the sensor measurement.

The previous section explained the cost function of the sensor optimization problem. This section extends the optimization problem by the constraints.

Clearly, the maximal length of the sensor must not exceed the machine component's length, and it must not fall below the lower bound $l_{f} L_{C}$, which is a small multiple of the component length $L_{C}$. This lower bound prevents a high model error due to the loss of information for noncaptured machine tool regimes.

Besides the sensor lengths, the possible sensor positions is constrained to the machine surface. During the design phase, it is allowed to place the sensor everywhere. Therefore the best possible positioning from that consideration is expected.

Afterwards the sensors are (virtually) placed at the expert's position to start at a useful initial value. Other useful starting values are the centers of the surfaces, or even solutions from previous positioning. Keep in mind that numerical methods often depend on the initial values to obtain a fast convergence. This time the initial values correspond to the initial positioning, see Figure 3 . Whereas the optimization criterion is the maximal squared axis length, the figures depict $S=\sqrt{\max \left(\lambda_{i, 1}, \lambda_{i, 2}, \lambda_{i, 3}\right)}$ the length of the longest axis in every point in the work space.

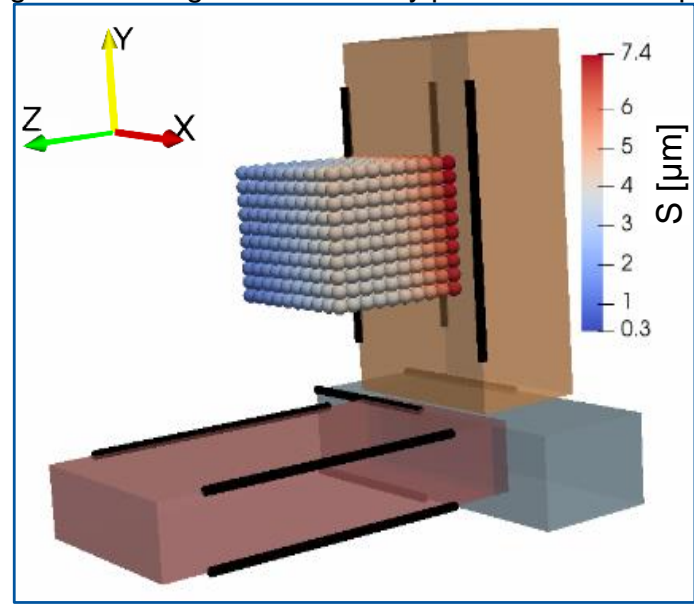

Fig. 3: Initial expert sensor positioning in design phase. Every sphere represents one point in the workspace.

Afterwards the optimization is performed with the component length factors $l_{f}=0.3$ and $l_{f}=0.7$ to obtain the maximal roots of the eigenvalues as depicted in the Figures 4 and 5 respectively. Interestingly, the optimization always led to the minimal possible sensor 
length for all rods. The fixed sensor length are used to prevent a minimization of the model error by reducing the length-dependent sensor uncertainty.

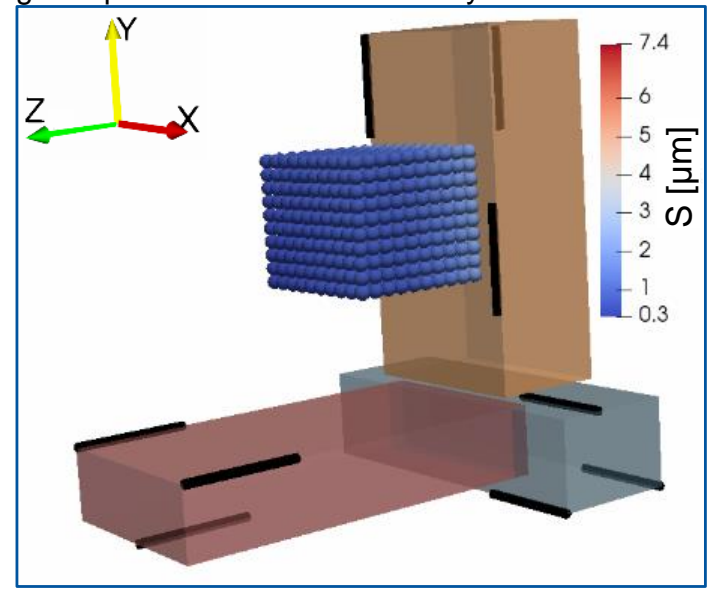

Fig. 4: Robust optimal sensor positioning with If $=0.3$ with mathematical approach

All three figures use the same color scale for easier visual comparison. Clearly the initial positioning has the largest domain with red spheres. This indicates a quite large optimization criterion and can be interpreted as the longest covariance ellipsoid axes respectively TCP uncertainty. Furthermore the smaller sensor length leads to the (theoretically) smallest covariance ellipsoids, hence to the most accurate prediction of the TCP error.

As the uncertainty of the prediction model is dependent on the IDS length, the authors assume the optimization method minimizes the IDS length in order to reduce the prediction uncertainty.

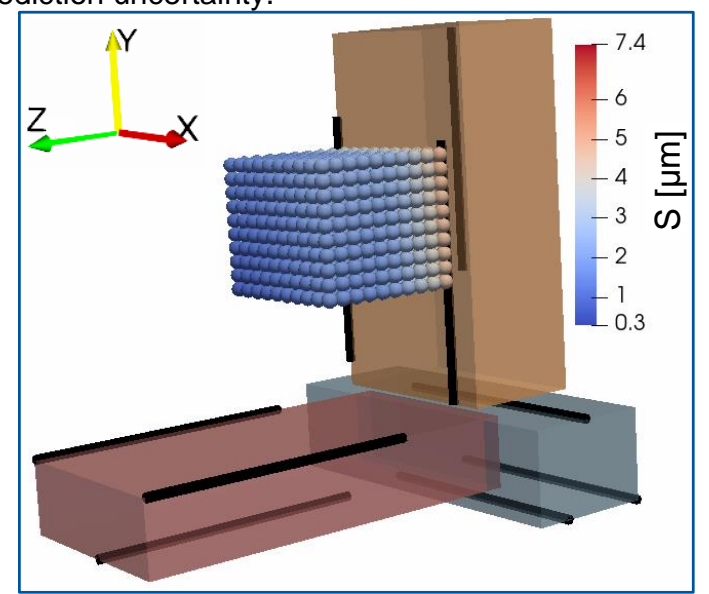

Fig. 5: Robust optimal sensor positioning with If $=0.7$ with mathematical approach

\subsection{Retrofitting the IDS to the surface}

This section focusses the retrofit of the IDS. In this case suitable and accessible mounting positions are needed. This adds several constraints:

- Parts of the surface are not accessible at all.

- Parts of the surface have no direct connection to the machine structure and therefore are not useful for direct displacement measurement (e. g. hoods).

- Some surfaces of the machine are occupied by cables, piping and other equipment. Mounting the sensors there is not possible or too time consuming.

These additional constraints have to be incorporated into the optimization strategy. Furthermore the positioning of the sensors is limited to the surface of the machine which implies geometric constraints. The correct identification of the available surfaces is additionally restricted by the accessibility of the surfaces. It can be limited by e. g. hoods or mounting points cannot be accessed with the necessary tools. The limited installation space, especially in the lower parts of the machine tool are depicted in Fig. 6. The available surfaces are colored.

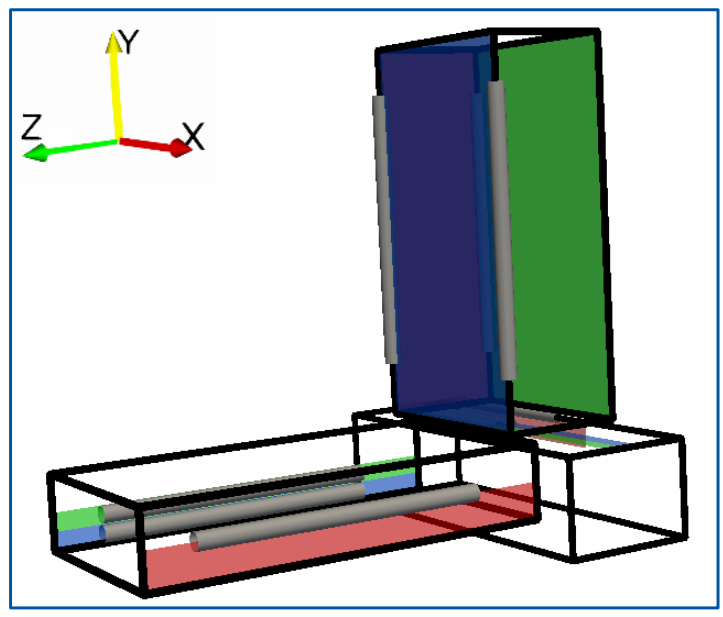

Fig 6: Limited installation space (colored)

\section{RESULTS AND COMPARISON}

In this section, the thermally induced TCP error is predicted based on the experience-based sensor positioning and the mathematical-based optimal sensor positioning for a specific thermal axis load. The results are then compared with the measured TCP error. All other parameters of the prediction model remained constant for both predictions, so the difference arises from the sensor positioning only.

For this purpose, IDS for both positioning methods were installed on the machine tool demonstrator, which is a horizontal-spindle machining center with a tCYXbZBw kinematic according to ISO10791-6 [ISO 1998]. To enable the parallel installation minor modifications were made for both placments. The resulting covariances respectively uncertainties of the installed setups are depicted in Fig. 7.

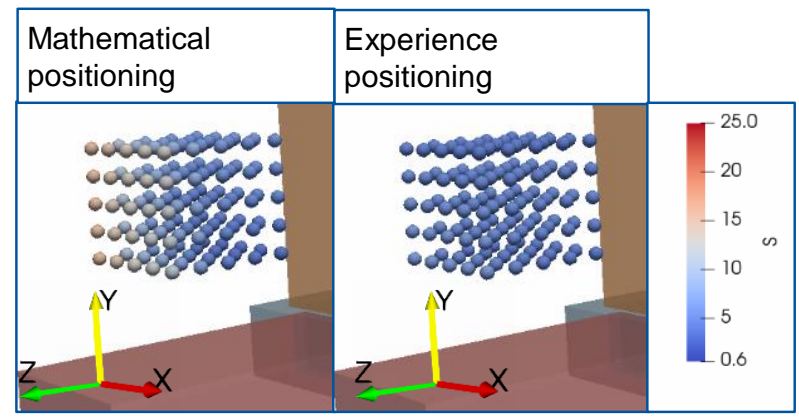

Fig. 7: Results for the retrofit of the IDS of the demonstrator machine

The advantage of the mathematical placement of lower covariances respectively uncertainties is not given for this case of a retrofit. The authors attribute this to the very little installation space limiting the freedom of the optimization. In addition, the definition of the accessible surfaces was highly iterative and the installation process was quite difficult, as many surfaces were not available.

An exemplary photo of the parallel installation is depictet in Fig. 8 together with an assignment of the location of installation. 


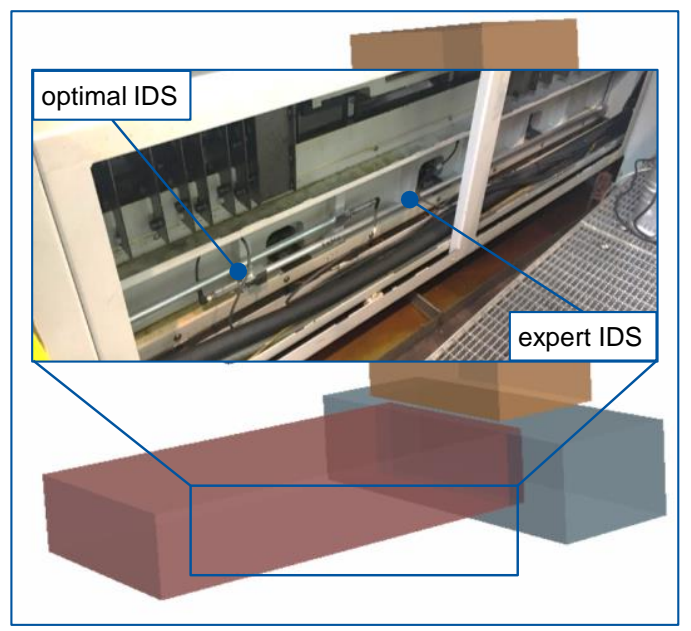

Fig. 8: Exemplary IDS installation

The linear motion of its $\mathrm{X}$-axis was selected as thermal load, as axis loads cause relevant TCP errors. Further thermal loads will be investigated in the future. The test cycle consists of two periods of time: 24 hours of axis cycling with a feed rate of $17.500 \mathrm{~mm} / \mathrm{min}$ and 24 hours of cooling down due to standstill. As designated in the [ISO 2007] norms, the machine's dives control remained enabled for more than 24 hours before the experiment, giving sufficient time to the structure to reach a steady state before the load cycle started.

A static R-test equipment was used for the continuous recording of the TCP error during the experiment [Weikert 2004]. The relative displacement between the measurement head of the R-test equipment and the reference ball mounted on the load unit on top of the work piece table could be measured in parallel to the axes of travel of the machine, see Fig. 9. As a result, the tilt and the rotation around the $X$ and $Y$ could not be estimated. In practical terms, this allows to evaluate the effect on the prediction of the positioning accuracy. Measurements of translational errors of several points in the work space where conducted. The most significant results are presented here. A further step, including the tilt and rotation errors is part of ongoing work. The position of the measurement setup is at $X-141$ Y 609 Z $500 \mathrm{~mm}$ in machine coordinates. The setup is depicted in Fig. 9.

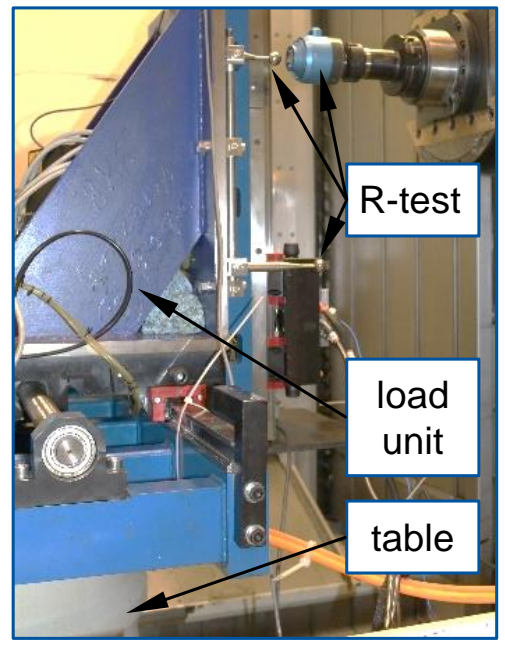

Fig. 9: R-test Measurement setup

The machine door was closed during the experiment, in order to isolate the R-test equipment from the ambient conditions of the workshop. Despite of its relatively large size $(850 \times 700 \times 800 \mathrm{~mm})$, the demonstrator machine tool is closed by covers from all sides, even from the top. As a result, this machine tool needs significantly more time to heat up or cool down or get affected by the ambient in comparison to most modern middle-sized machine tools, which are often not covered up on top.

As recommended in Annex D in the ISO 230-3 norms, a low-pass filter was applied on the signals of the TCP error measurement. In addition, other mechanical and thermal effects with a higher frequency, such as the effect of opening the machine door for a few minutes, are not of interest for these investigations. Hence, a first order Butterworth filter with a cutoff-frequency corresponding to a period of 2 minutes was applied on the raw signal with a sampling rate of 1 second.

In order to estimate the measurement uncertainty of the applied R-test procedure in the described experiment, the following influences are considered:

a) uncertainty of gauging a reference ball $u_{p}$,

b) influence of temperature changes on the measurement equipment $u_{t}$ and

c) influence of temperature changes on the reference ball and its holder during the measurement $u_{b}$.

The uncertainty budget of the TCP error measurement is listed in Table 1. The first part $u_{p}$ has been determined by the manufacturer with $0.5 \mu \mathrm{m}$. The second term $u_{t}$ is also given by the manufacturer with a thermal expansion coefficient of $0.01 \% / \mathrm{K}$. Finally, the third part can be calculated using the thermal expansion coefficient of the work piece table material (cast iron $-11 \mu \mathrm{m} / \mathrm{m} / \mathrm{K}$ ), the load unit material (steel $-11 \mu \mathrm{m} / \mathrm{m} / \mathrm{K}$ ) and the reference ball holder material (invar steel $-1.2 \mu \mathrm{m} / \mathrm{m} / \mathrm{K}$ ) and the total length of $400 \mathrm{~mm}$. A temperature change of $1 \mathrm{~K}$ during the experiments can thus provoke up to $4.8 \mu \mathrm{m}$ of distance change. Considering the recommended divisors in [ISO/IEC 2008], the combined uncertainty is therefore $4,70 \mu \mathrm{m}$. The IDS uncertainty for the expert / optimized positioning is $2,9 \mu \mathrm{m} / 2,7 \mu \mathrm{m}(\mathrm{Z}), 2,6 \mu \mathrm{m} / 8,94 \mu \mathrm{m}(\mathrm{Y})$ respectively $3,8 \mu \mathrm{m} / 6,0 \mu \mathrm{m}(Z)$ for $95 \%$ confidence interval of the results of this chapter.

Tab. 1: Contributions to measurement uncertainty.

\begin{tabular}{ccccc}
\hline Source & Probing & Device & Ball & Combined \\
\hline Value & $1 \mu \mathrm{m}$ & $0.0004 \mu \mathrm{m}$ & $8,1 \mu \mathrm{m}$ & - \\
& & \multicolumn{2}{c}{ Rectangula } & Rectangul \\
Distribution & Normal & $\mathrm{r}$ & $\mathrm{ar}$ & - \\
Divisor (k) & 2 & $\sqrt{ } 3$ & $\sqrt{ } 3$ & - \\
Sensitivity & 1 & 1 & 1 & - \\
Uncertainty & $0.5 \mu \mathrm{m}$ & $0.00023 \mu \mathrm{m}$ & $4.67 \mu \mathrm{m}$ & $4.70 \mu \mathrm{m}$ \\
Symbol & $\mathrm{U}_{\mathrm{p}}$ & $\mathrm{u}_{\mathrm{t}}$ & $\mathrm{U}_{\mathrm{b}}$ & $\mathrm{u}_{\mathrm{c}}$ \\
Method & measured & estimated & estimated & estimated \\
\hline
\end{tabular}

The work piece table (not depicted in the figures) was also taken into account in this estimation, because this machine component was not included in the sensor positioning. The purpose of these experimental investigations is to compare the measured TCP error with the prediction based on two sensor positionings. Since none of them contains any information about the work piece table, it is suitable to include this component's behavior as part of the measurement uncertainty. This way, the estimated measurement uncertainty contains all effects that the prediction cannot take into account. The combined measurement uncertainty is not depicted in Fig. 10 and Fig. 11, in order to keep the clarity of the diagrams intact. Since the measurement uncertainty of the IDS is 
dependent on the true thermal expansion coefficient of the used CFRP rods, a longer IDS is automatically related to a higher measurement uncertainty. The mathematicalbased sensor positioning contains IDS with a length of $715 \mathrm{~mm}$, while the experienced based sensor positioning includes IDS with lengths ranging from $968 \mathrm{~mm}$ to 1.936 $\mathrm{mm}$. The method used to derive the prediction uncertainty of the model due to uncertainty propagation from the IDS is explained in [Riedel 2017].

The prediction of the experience based sensor positioning $\mathrm{Pe}_{\mathrm{e}}$ is compared with the measured TCP error in Fig. 10. The prediction with the mathematical based sensor positioning $\mathrm{P}_{\mathrm{m}}$ is compared with the measured TCP error in Fig. 11. The shaded area between the measured and the predicted TCP error is drawn to facilitate the comparison. It is evident that the prediction $\mathrm{P}_{\mathrm{m}}$ follows the trend of the measured TCP error in Z-direction more consistently than the prediction $\mathrm{Pe}$, although not so consistently in X-direction.

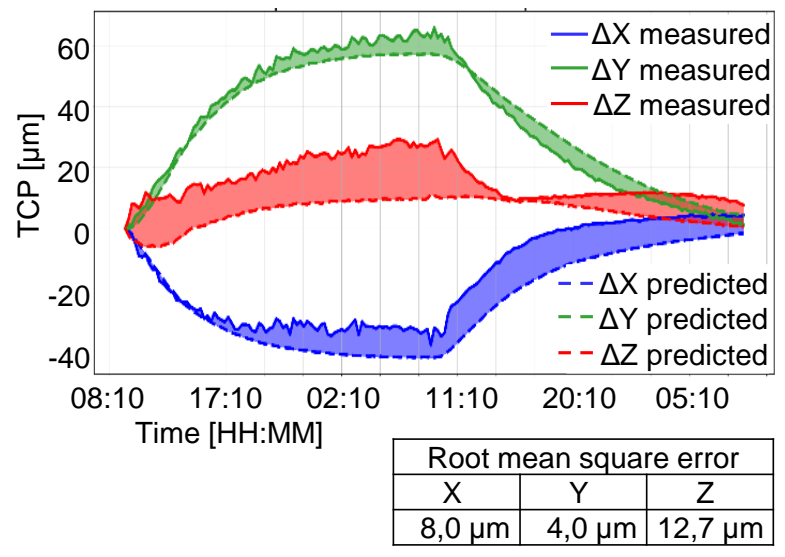

Fig. 10: Comparison of measured TCP error with the TCP error predicted by the experience-based sensor positioning, as depicted in Figure 3.

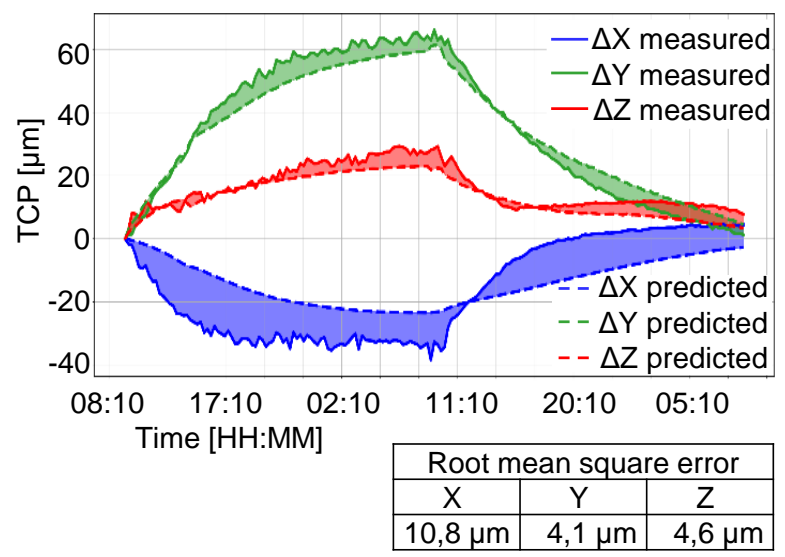

Fig. 11: Comparison of measured TCP error with the

TCP error predicted by mathematical-based optimal sensor positioning, as depicted in Figure 5

A more detailed investigation of the predicted axis error components that leads to the prediction of the overall TCP error indicated that the discrepancies of both methods originate from angular errors.

In summary, a worse prediction of the overall TCP error was not by either of the two sensor positioning methods was not a result of missing information, but one of false interpretation. It is also evident that $\mathrm{Pe}_{\mathrm{e}}$ was better at the prediction of the $\mathrm{X}$-axis, located at the rear side of the machine base, while $\mathrm{P}_{\mathrm{m}}$ was better at the prediction of the
Y-axis, located at the moving column. As a consequence, the mathematical-based optimal sensor positioning has led to better results and the expected smaller uncertainties of the prediction model, but not for all components of the demonstrator machine tool investigated in this paper.

Another significant factor to consider is that this test was executed only on one point in working space. Since the expected improvement of the mathematical sensor positioning is also dependent on the working space location, a repetition of the presented experiment with measurement that comprises angular errors as well can provide more insights to compare the two sensor positioning methodologies.

\section{SUMMARY AND OUTLOOK}

In conclusion, the proposed sensor positioning methodologies exhibit the following properties:

a) The full potential of the mathematical approach to improve the prediction accuracy can be used during the design phase of a machine tool. In this case, the moderate effort required for the mathematical approach well fits the time frame of the design process.

b) The use of this method for a retrofit cannot be recommended with respect to the presented demonstrator machine. According to the authors opinion applications with larger available installation space could lead to an improvement in comparison to experience based sensor positioning, if they do not necessitate an iterative installation procedure to apply the optimal positioning..

c) The expert based approach is mainly focused on fast, but reliable results under the given installation conditions of the machine tool.

However, using shorter IDS has shown a good potential for some configurations presented in this paper. The reduction of the TCP uncertainty due to shorter IDS length poses the question what IDS length can be considered the optimal length.

Further investigations, especially including local heating effects, have to be performed, in order to get reliable results that validate the robustness of the prediction model under the most challenging conditions. The most difficult stress-test for any sensor positioning and prediction model based on IDS is a local heating of an axis, which is also the case for many manufacturing processes, in which one machine axis moves rapidly within a short stroke. Any local deformation is directly correlated with the rest of the deformation field, but the principle of the IDS is to measure the integral deformation throughout a specific length. Hence, local effects are the most difficult to derive. This topic is part of the ongoing IDS research.

\section{Acknowledgement}

Funded by the German Research Foundation Project-ID 174223256 - TRR 96 - Subprojects B06, B08 and $\mathrm{C} 03$.

\section{BIBLIOGRAPHY}

[Atkinson 1996] Atkinson A. C. The Usefulness of Optimum Experimental Designs. Journal of the Royal Statistical Society. Series B (Methodological). 1996, Vol. 58, pp. 59-76, ISSN: 00359246.

[Atkinson 2007] Atkinson A. C., Donev A. N. und Tobias R. D. Optimum Experimental Designs, with SAS. Oxford : 
Oxford University Press, 2007, Vol. 34, ISBN: 978-0-19929660-6.

[Brecher 2018] Brecher C. et al. Metrological analysis of a mechatronic system based on novel deformation sensors for thermal issues in machine tools. In: Procedia CIRP, Vol 77, Special Issue for the 8th CIRP HPC Conference 2018, pp. 517-520, ISSN 2212-8271

[Brecher 2020] Brecher C.et al. Evaluation of optimal positioning methods of integral deformation sensors for the correction of thermal errors in machine tools. In: C. Brecher, ed. Aachen, 2020. ISBN: 978-0-9957751-6-9

[Fletcher 2013] Fletcher, $R$ Practical Methods of Optimization, Wiley, 2013, ISBN: 9781118723203

[ISO 1998] ISO 10791-6 Test conditions for machining centres. - Part 6: Accuracy of feeds, speeds and interpolations, 1998

[ISO 2007] ISO 230-3 Test code for machine tools. - Part 3: Determination of thermal effects. 2007

[ISO/IEC 2008] ISO/IEC Guide 98-3 Uncertainty of measurement. - Joint Committee for guides in Metrology - Bd. Part 3: Guide to the expression of uncertainty in measurement, 2008

[Liu 2020] Liu Yunsheng [et al.] Robust machine tool thermal error compensation modelling based on temperature-sensitive interval segmentation modelling technology, Int J Adv Manuf Technol . - 2020. - Bd. 106.

[Riedel 2017] Riedel Mirko et al. Funktionsprinzip der messtechnisch basierten Korrektur thermischer Verlagerungen am Versuchsträger MAX. In: C. Brecher, ed. 5. Kolloq. SFB / TR96. Chemnitz, 2017.ISBN 978-386780-516-2

[Tan 20] Tan Feng et al. A wrapper approach-based key temperature point selection and thermal error modeling method. Int. Journal of Advanced Manufacturing Technol., 2020. Vol. 106, pp. 907-920. ISSN 1433-3015

[Wachter 2002] Wachter Andreas, An Interior Point Algorithm for Large-Scale Nonlinear Optimization with Applications in Process Engineering. PhD Thesis, Carnegie Mellon University, Pittsburgh, USA, 2002

[Wei 2018] Wei Xian et al. Study on optimal independent variables for the thermal error model of CNC machine tools. Int. Journal of Advanced Manufacturing Technol., 2018. Vol. 98, pp.657-669. ISSN 1433-3015

[Zhou 2019] Zhou Zude et al. The selection of key temperature measurement points for thermal error modeling of heavy-duty computer numerical control machine tools with density peaks clustering. Advances in Mechanical Engineering, 2019, Vol 11, No. 4, pp.1-11, ISSN 1687-814 